\title{
Type of dyslipidemia and achievement of the LDL-cholesterol goal in chronic kidney disease patients at the University Hospital
}

This article was published in the following Dove Press journal:

Vascular Health and Risk Management

4 November 2015

Number of times this article has been viewed

\section{Tamon Sangsawang \\ Apiradee Sriwijitkamol}

Division of Endocrinology and Metabolism, Department of Medicine, Faculty of Medicine Siriraj Hospital, Mahidol University, Bangkok, Thailand
Correspondence: Apiradee Sriwijitkamol Division of Endocrinology and Metabolism, Department of Medicine, 8th floor Asadang building, Faculty of Medicine Siriraj Hospital, Mahidol University, Prannok street, Bangkok-noi, Bangkok, Thailand 10700

Tel +66 24197799

Fax +66 24197792

Email apiradeesi99@hotmail.com
Background: Chronic kidney disease (CKD) has been defined as a coronary artery disease risk equivalent. Therefore, the current guideline has been recommended for CKD patients to reach and maintain a low-density lipoprotein-cholesterol (LDL-C) goal of less than $100 \mathrm{mg} / \mathrm{dL}$. However, the data regarding the achievement of LDL-C goal in these patients is lacking.

Objective: This study was conducted to evaluate the types of dyslipidemia affecting patients with CKD stages 3 and 4 and to determine whether these patients achieved LDL-C goal.

Methods: We performed a retrospective chart review of patients with CKD stage 3 or 4 and dyslipidemia who were followed-up at Siriraj Hospital between October 2011 and September 2012.

Results: In total, 150 patients with CKD stage 3 or 4 and dyslipidemia were recruited. The mean age was $72 \pm 10$ years, and the body mass index was $25.6 \pm 4 \mathrm{~kg} / \mathrm{m}^{2} ; 60 \%$ had CKD stage 3 with an estimated glomerular filtration rate of $34 \pm 12 \mathrm{~mL} / \mathrm{min} / 1.73 \mathrm{~m}^{2}$, and $54 \%$ had type 2 diabetes. The percentage of patients with hypercholesterolemia was $78 \%$, hypertriglyceridemia $54 \%$, and low high-density lipoprotein-C $36 \%$. Of these, $52 \%$ had mixed hyperlipidemia. Statin treatment was prescribed to $87 \%$ of the patients, of which only $31.3 \%$ achieved the LDL-C goal according to the National Cholesterol Education Program and the European Society of Cardiology/ European Atherosclerosis Society recommendations. Patients who did not achieve the LDL-C goal had a higher cholesterol level at diagnosis and higher prevalence of type 2 diabetes and stroke than those who achieved it.

Conclusion: Two-thirds of CKD patients with hyperlipidemia had mixed hyperlipidemia. Despite the high frequency of statin treatment, only one-third of patients with CKD achieved the LDL-C goal. Thus, a developmental plan for the management of dyslipidemia in patients with CKD should be implemented to increase their achievement of the LDL-C goal.

Keywords: chronic kidney disease, hyperlipidemia, LDL-C, dyslipidemia

\section{Introduction}

Chronic kidney disease (CKD) is a common health issue, and its incidence may continue to increase worldwide given the rise in type 2 diabetes and other CKD risk factors. ${ }^{1}$ Data from the Thai Screening and Early Evaluation of Kidney Disease study ${ }^{2}$ demonstrated that CKD prevalence in the Thai population increased compared with the incidence reported in a previous study (17.5\% vs 4.6\%-13.8\%, respectively). Among Thai patients with CKD, 27.5\% had hypertension and $11.9 \%$ had type 2 diabetes, which were the two main causes of CKD. ${ }^{2}$ A previous study ${ }^{3}$ has shown the association between the reductions of estimated glomerular filtration rate (eGFR) and the increase of cardiovascular events and accelerated atherosclerosis, which result in increased morbidity and mortality. Prevention and treatment of cardiovascular disease are major 
considerations in the management of individuals with CKD. ${ }^{4}$ Another recent study ${ }^{5}$ showed that lowering cholesterol can decrease cardiovascular morbidity and mortality in CKD patients. Therefore, the National Cholesterol Education Program (NCEP) ${ }^{6}$ and the European Society of Cardiology/ the European Atherosclerosis Society ${ }^{7}$ have defined CKD as a coronary artery disease risk equivalent and recommend a low-density lipoprotein-cholesterol (LDL-C) goal of 100 and $70 \mathrm{mg} / \mathrm{dL}$ for CKD patients, respectively. However, data regarding the type of dyslipidemia in CKD patients in Thailand is lacking, especially for patients with CKD stages 3 and 4 who have marked impairment of eGFR. Therefore, this study was conducted to evaluate the types of dyslipidemia that affect patients with CKD stages 3 and 4 and to determine whether the LDL-C goal was achieved in these patients.

\section{Materials and methods Study population}

Patients who had CKD stage 3 or 4 and dyslipidemia, according to International Classification of Diseases 10 (N18.9) criteria, and were followed-up for at least 1 year in the internal medicine outpatient department (OPD) at Siriraj Hospital between October 1, 2011 and September 30, 2012, were recruited to allow sufficient time for the health care provider to adjust the lipid-lowering agent. In this study, eGFR was calculated using the National Kidney Foundation Chronic Kidney Disease Epidemiology Collaboration equation and eGFR calculator program. CKD stages 3 and 4 were defined as the estimated eGFR $30-59 \mathrm{~mL} / \mathrm{min} / 1.73 \mathrm{~m}^{2}$ and $15-29 \mathrm{~mL} / \mathrm{min} / 1.73 \mathrm{~m}^{2}$, respectively. Patients at other stages of $\mathrm{CKD}$, those who had received the lipid-lowering agents before attending OPD at Siriraj Hospital and those who did not have lipid profiles at the time of diagnosis and at the last visit to the internal medicine OPD were excluded. The Siriraj Institutional Review Board approved the study, which was conducted in compliance with the Declaration of Helsinki. Based on the type of study, the Siriraj Institutional Review Board waived the need for formal informed consent.

\section{Data collection and outcome measurements}

The computer-based data of each patient were reviewed. Data were extracted as follows. We obtained the baseline and laboratory characteristics, including age, sex, body mass index, eGFR, CKD stage, comorbidities, type and dosage of cholesterol-lowering agent used, and lipid profile at the time of diagnosis and at the last visit to the internal medicine OPD.
The definition of dyslipidemia was categorized as follows: hypercholesterolemia was defined as cholesterol level greater than $200 \mathrm{mg} / \mathrm{dL}$ and/or LDL-C level greater than $100 \mathrm{mg} / \mathrm{dL}$; hypertriglyceridemia, as triglyceride level greater than $150 \mathrm{mg} / \mathrm{dL}$; and low high-density lipoprotein-cholesterol (HDL-C) lower than $40 \mathrm{mg} / \mathrm{dL}$ in men and $50 \mathrm{mg} / \mathrm{dL}$ in women. Patients who had more than one abnormal lipid component were defined as having mixed hyperlipidemia. Statin potency was classified as low, moderate, and high according to the 2013 the American College of Cardiology/ American Heart Association (ACC/AHA) Blood Cholesterol Guideline. ${ }^{8}$ In brief, the low-potency statin was simvastatin $10 \mathrm{mg}$. The moderate-potency statins included simvastatin 20-40 mg, atorvastatin 10-20 mg, and rosuvastatin 5-10 mg. The high-potency statins included simvastatin, atorvastatin, and rosuvastatin at doses higher than 40, 20, and $10 \mathrm{mg}$, respectively. Add-on ezetimibe to a moderate-potency statin was also considered as high potency.

The achievement of the LDL-C goal according to the NCEP Adult Treatment Panel III recommendation was evaluated using the lipid levels at the last visit to the internal medicine OPD. For patients with CKD and diabetes or atherosclerotic diseases, such as peripheral arterial disease, abdominal aortic aneurysm, symptomatic carotid artery stenosis, stroke, and coronary artery disease, the LDL-C goal was $70 \mathrm{mg} / \mathrm{dL}$, whereas for patients with CKD without comorbidities, the LDL-C goal was $100 \mathrm{mg} / \mathrm{dL}$. The "achieved LDL-C goal" group consisted of patients with an LDL-C level lower than the goal LDL-C and the "did not achieve LDL-C goal" group consisted of patients with an LDL-C greater than the goal LDL-C. Factors associated with achieving the LDL-C goal were then evaluated.

\section{Statistical analysis}

Statistical analysis was carried out with SPSS software, version 18 (SPSS Inc., Chicago, IL, USA). Continuous data were expressed as mean \pm standard deviation or median (min, max) as appropriate. Comparisons between groups were analyzed by the Student's $t$-test. Categorical data were expressed as percentages. Comparisons were analyzed by the chi-square test or Fisher's exact test. Statistical significance was declared if the $P$-value was less than 0.05 .

\section{Results \\ Baseline characteristics}

In total, 150 patients diagnosed with CKD stage 3 or 4 and dyslipidemia were included in this study. The characteristics of the subjects are described in Table 1. The patients 
Table I Clinical and laboratory characteristics

\begin{tabular}{ll}
\hline Characteristics & $\mathbf{N}=\mathbf{I 5 0}$ \\
\hline Age (years) & $72 \pm 10$ \\
Male & $96(64.0)$ \\
BMI $\left(\mathrm{kg} / \mathrm{m}^{2}\right)$ & $25.6 \pm 4$ \\
eGFR $\left(\mathrm{mL} / \mathrm{min} / \mathrm{I} .73 \mathrm{~m}^{2}\right)$ & $34 \pm 12$ \\
CKD stage & \\
CKD stage 3 & $90(60.0)$ \\
CKD stage 4 & $60(40.0)$ \\
Comorbid diseases & \\
Hypertension & $137(91.3)$ \\
Type 2 diabetes & $81(54.0)$ \\
Coronary artery disease & $27(18.0)$ \\
Stroke & $25(16.7)$ \\
Abdominal aortic aneurysm & $4(2.7)$ \\
Peripheral arterial disease & $3(2.0)$ \\
\hline
\end{tabular}

Note: Data are presented as mean \pm SD or $n(\%)$ as appropriate.

Abbreviations: BMI, body mass index; CKD, chronic kidney disease; eGFR, estimated glomerular filtration rate; SD, standard deviation; min, minute.

had a mean age of $72 \pm 10$ years and a body mass index of $25.6 \pm 4 \mathrm{~kg} / \mathrm{m}^{2}$. Ninety patients $(60 \%)$ had CKD stage 3 and a mean eGFR of $34 \pm 12 \mathrm{~mL} / \mathrm{min} / 1.73 \mathrm{~m}^{2}$. Sixty-four percent of the patients were male and $54 \%$ had type 2 diabetes. Seventyeight percent of these patients had hypercholesterolemia, $54 \%$ had hypertriglyceridemia, and 36\% had a low HDL-C level. Of these, $52 \%$ had mixed hyperlipidemia. Fifty of 150 patients had a target LDL goal of less than $100 \mathrm{mg} / \mathrm{dL}$, whereas 100 of 150 had a target LDL goal of less than $70 \mathrm{mg} / \mathrm{dL}$, according to the NCEP. ${ }^{6}$ Eighty-seven percent of the patients were being treated with statins, $5 \%$ with a combination of statin and ezetimibe, and $3 \%$ with fibrates

Table 2 Lipid level and lipid lowering at the outpatient department

\begin{tabular}{lll}
\hline Lipid level & $\begin{array}{l}\text { At diagnosis } \\
(\mathbf{m g} / \mathbf{d L})\end{array}$ & $\begin{array}{l}\text { At the last visit } \\
(\mathbf{m g} / \mathbf{d L})\end{array}$ \\
\hline Cholesterol & $22 \mathrm{I} \pm 6 \mid$ & $174 \pm 35$ \\
Triglycerides & $186 \pm I \mathrm{II}$ & $138 \pm 7 \mid$ \\
HDL-C & $47 \pm 13$ & $52 \pm 16$ \\
LDL-C & $134 \pm 48$ & $95 \pm 29$ \\
Lipid-lowering treatment; $\mathrm{n}(\%)$ & \\
$\quad$ No treatment & $18(12.0)$ & \\
Statin treatment & $130(86.7)$ & \\
Fibrate treatment & $4(2.7)$ & \\
Statin + fibrate & $2(1.3)$ & \\
Statin + ezetimibe & $7(4.7)$ & \\
Potency of statin; $\mathrm{n}(\%)$ & & \\
Low potency & $55(36.7)$ & \\
Moderate potency & $65(43.3)$ & \\
High potency & $10(6.7)$ & \\
\hline
\end{tabular}

Note: Data are presented as mean \pm SD.

Abbreviations: HDL-C, high-density lipoprotein-cholesterol; LDL-C, low-density lipoprotein-cholesterol; SD, standard deviation.
(Table 2). The mean lipid levels at the time of diagnosis and at the last visit to the OPD are shown in Table 2. Most of the subjects were treated with low- and moderate-potency statins ( $37 \%$ and $43 \%$, respectively).

\section{Achievement of LDL-C goal}

Only 47 patients (31.3\%) achieved the LDL-C goal according to the NCEP recommendation. As shown in Table 3, a greater proportion of patients in the "did not achieve LDL-C" group had comorbidities, including type 2 diabetes and stroke, compared with the patients in the "achieved LDL-C" group; therefore, in this group, the LDL-C goal for the former should

Table 3 Comparison of clinical and laboratory characteristics between the "achieved LDL-C goal" and "did not achieve LDL-C goal" groups

\begin{tabular}{|c|c|c|c|}
\hline Characteristics & $\begin{array}{l}\text { Achieved } \\
\text { LDL-C goal }\end{array}$ & $\begin{array}{l}\text { Did not achieve } \\
\text { LDL-C goal }\end{array}$ & $P$-value \\
\hline N (\%) & $47(31.3)$ & $103(68.7)$ & \\
\hline Age (years) & $7 I \pm 11$ & $72 \pm 9$ & 0.448 \\
\hline Male, n (\%) & $30(63.8)$ & $66(64.1)$ & 0.977 \\
\hline BMI $\left(\mathrm{kg} / \mathrm{m}^{2}\right)$ & $25 \pm 3$ & $26 \pm 5$ & 0.44 \\
\hline eGFR $\left(\mathrm{mL} / \mathrm{min} / \mathrm{l} .73 \mathrm{~m}^{2}\right)$ & $34.1 \pm 12.0$ & $33.8 \pm 12.0$ & 0.903 \\
\hline \multicolumn{3}{|l|}{ CKD stage, $\mathrm{n}(\%)$} & 0.22 \\
\hline CKD stage 3 & $30(63.8)$ & $60(57.3)$ & \\
\hline CKD stage 4 & $16(34.0)$ & $44(42.7)$ & \\
\hline $\begin{array}{l}\text { LDL-C goal of less than } \\
70 \mathrm{mg} / \mathrm{dL}, \mathrm{n}(\%)\end{array}$ & $17(36.2)$ & $83(80.6)$ & $<0.001$ \\
\hline $\begin{array}{l}\text { LDL-C goal of less than } \\
100 \mathrm{mg} / \mathrm{dL}, \mathrm{n}(\%)\end{array}$ & $30(63.8)$ & $20(19.4)$ & $<0.001$ \\
\hline Statin treatment, $\mathrm{n}(\%)$ & $4 \mid(87.2)$ & $89(86.4)$ & 0.89 \\
\hline \multicolumn{4}{|l|}{ Comorbid diseases, n (\%) } \\
\hline Type 2 diabetes & $10(2 \mathrm{I} .3)$ & $71(68.9)$ & $<0.005$ \\
\hline Hypertension & $42(89.4)$ & $95(92.2)$ & 0.562 \\
\hline $\begin{array}{l}\text { Coronary artery } \\
\text { disease }\end{array}$ & $8(17.0)$ & $19(18.4)$ & 0.833 \\
\hline Stroke & $3(6.4)$ & $22(2 \mathrm{I} .4)$ & 0.022 \\
\hline $\begin{array}{l}\text { Abdominal aortic } \\
\text { aneurysm }\end{array}$ & $2(4.3)$ & $2(1.9)$ & 0.415 \\
\hline $\begin{array}{l}\text { Peripheral arterial } \\
\text { disease }\end{array}$ & $2(4.3)$ & $\mathrm{I}(\mathrm{I} .0)$ & 0.183 \\
\hline \multicolumn{4}{|l|}{ Baseline lipid level (mg/dL) } \\
\hline Cholesterol & $205 \pm 56$ & $228 \pm 62$ & 0.034 \\
\hline LDL-C & $120 \pm 46$ & $142 \pm 47$ & 0.023 \\
\hline HDL-C & $46 \pm 13$ & $47 \pm 13$ & 0.752 \\
\hline Triglycerides & $179 \pm 124$ & $189 \pm 107$ & 0.666 \\
\hline \multicolumn{4}{|c|}{ Lipid level at time of study $(\mathrm{mg} / \mathrm{dL})$} \\
\hline Cholesterol & $142 \pm 20$ & $|88 \pm 3|$ & $<0.005$ \\
\hline LDL-C & $71 \pm 16$ & $107 \pm 28$ & $<0.005$ \\
\hline HDL-C & $53 \pm 18$ & $52 \pm 16$ & 0.644 \\
\hline Triglycerides & $120 \pm 76$ & $146 \pm 69$ & 0.088 \\
\hline
\end{tabular}

Note: Data are presented as mean \pm SD.

Abbreviations: BMI, body mass index; CKD, chronic kidney disease; eGFR, estimated glomerular filtration rate; HDL-C, high-density lipoprotein-cholesterol; LDL-C, low-density lipoprotein-cholesterol; SD, standard deviation; min, minute. 
be lower than that for the "achieved LDL-C" group. These patients also had a higher cholesterol level at the time of diagnosis than those in the "achieved LDL-C" group. Although the "did not achieve LDL-C" group had higher cholesterol levels at baseline and the LDL-C goal should have been lower, it would be expected that the "did not achieve LDL-C" group would have required treatment with statins of higher potency. However, the potency of statins used in these two groups was not significantly different as shown in Figure 1.

\section{Discussion}

In the present study, approximately half of the patients with CKD stage 3 or 4 and dyslipidemia had mixed hyperlipidemia. These findings are consistent with the results of a previous study ${ }^{7}$ in which most patients with CKD stage 3-5 had mixed dyslipidemia. These data have important clinical implications because patients with mixed dyslipidemia require combination treatment with statins and fibrates for the management of their abnormal lipid level. These two types of drugs have significant drug interactions, ${ }^{9}$ and thus, clinicians who manage these patients should be cautious when prescribing such medications.

According to our results, $87 \%$ of the patients received statin treatment. Despite the high frequency of statin treatment, only $31.3 \%$ of the patients achieved the LDL-C goal. According to our results, patients whose LDL-C goal should have been lower, and who required treatment with statins of higher potency, received statins of low-to-moderate potency. We hypothesized that the following were possible reasons why these patients did not achieve the LDL-C goals: limited knowledge of clinicians, fear of statin-related side effects led to poor treatment adherence, and the goal of LDL-C in patients with comorbidities was markedly low, especially for patients with type 2 diabetes.

Despite the fact that CKD has been known to be a coronary artery disease risk equivalent since $2004,{ }^{10}$ no previous studies

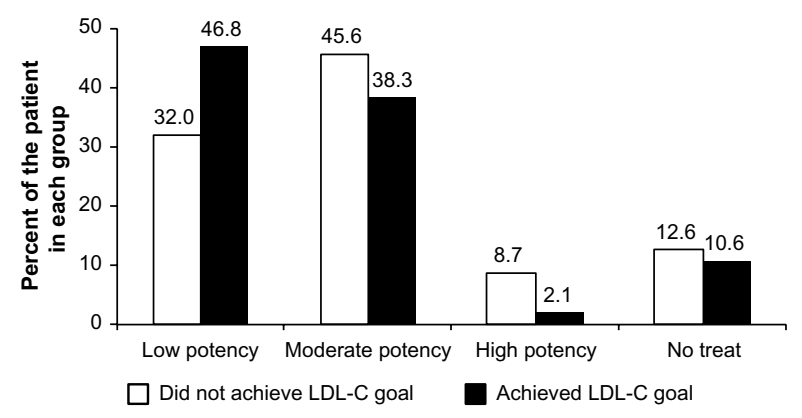

Figure I Comparison of the potency of statins used for patients who did not achieve the LDL-C goal (open bar) and those who achieved the LDL-C goal (solid bar). Abbreviation: LDL-C, low-density lipoprotein-cholesterol. had demonstrated that lowering cholesterol could reduce cardiovascular morbidity and mortality among these patients until recently. ${ }^{11}$ It was recently demonstrated that reduction of LDL-C with simvastatin plus ezetimibe could safely reduce the incidence of major atherosclerotic events in a wide range of patients with advanced CKD. Moreover, recent meta-analysis demonstrated that $\mathrm{CKD}$ patients could benefit from statin treatment in terms of cardiovascular events, mortality, and renal outcomes. ${ }^{12}$ General practitioners may not be aware of the benefit of lowering LDL-C in these patients. Thus, a developmental program should be launched to include updated clinical policies and treatment guidelines for patients with dyslipidemia with various comorbidities, especially CKD.

Based on our study results, patients who did not achieve the LDL-C goal had a higher cholesterol level at baseline and had comorbidities for which a lower LDL-C goal should be established; therefore, these patients should have been treated with statins at a high dose or needed high-potency statins to achieve the LDL-C goal. The adverse effects of lipid-lowering drugs, including myositis and hepatitis, are commonly found in patients with advanced CKD, particularly those exposed to a high statin dose or potency. Therefore, it is likely that clinicians prescribed a low dose or a statin of low-to-moderate potency to prevent or ameliorate these adverse effects, which could explain the lack of achievement of the LDL-C goal in this group of patients.

The main limitation of this study was its retrospective design, which involved the review of computer-based patient data. Therefore, we did not have data on the duration of statin treatment. We did not know whether the health care provider adjusted the patient treatment regimens. However, we recruited only the patients who had been in follow-up at the OPD for at least 1 year. We considered that this was sufficient time for the health care provider to adjust the regimens. Although the Kidney Disease Improving Global Outcomes organization developed and released a clinical practice guideline that includes recommendations of statin doses for patients with $\mathrm{CKD},{ }^{13}$ further study should be performed to evaluate the dose and type of statin treatment for these patients.

\section{Conclusion}

Among CKD patients with hyperlipidemia, approximately two-thirds of the patients had mixed hyperlipidemia. Despite the high frequency of statin treatment, only one-third of CKD patients achieved the LDL-C goal. The high level of cholesterol at diagnosis and low LDL-C goal, such as that observed for patients with type 2 diabetes and stroke were probably the main factors leading to failure to achieve the 
LDL-C goal. Thus, a developmental plan for the management of dyslipidemia in CKD patients should be launched to increase the percentage of patients who achieve the LDL-C goal, which could result in decreased cardiovascular morbidity and mortality among these patients.

\section{Acknowledgments}

This work was supported by grants from the Siriraj Grant for Research Development, Faculty of Medicine Siriraj Hospital, Mahidol University, Bangkok, Thailand (AS). The authors would like to thank Ms Kemjira Karngateklang for her assistance with the statistical analysis.

\section{Author contributions}

TS contributed to acquisition of data, performed the statistical analysis and data interpretation, and was involved in drafting the manuscript. AS contributed to the conception of the study, participated in its design, was involved in revising the manuscript, and approved this version for publication. All authors read and approved the final paper.

\section{Disclosure}

The authors report no conflicts of interest in this work.

\section{References}

1. Muralidharan A, White S. The need for kidney transplantation in lowand middle-income countries in 2012: an epidemiological perspective. Transplantation. 2015;99(3):476-481.

2. Ingsathit $\mathrm{A}$, Thakkinstian $\mathrm{A}$, Chaiprasert $\mathrm{A}$, et al. Prevalence and risk factors of chronic kidney disease in the Thai adult population: Thai SEEK study. Nephrol Dial Transplant. 2010;25(5):1567-1575.
3. Schiffrin EL, Lipman ML, Mann JF. Chronic kidney disease: effects on the cardiovascular system. Circulation. 2007;116(1):85-97.

4. Go AS, Chertow GM, Fan D, McCulloch CE, Hsu CY. Chronic kidney disease and the risks of death, cardiovascular events, and hospitalization. N Engl J Med. 2004;351(13):1296-1305.

5. Kanbay M, Turgut F, Covic A, Goldsmith D. Statin treatment for dyslipidemia in chronic kidney disease and renal transplantation: a review of the evidence. J Nephrol. 2009;22(5):598-609.

6. National Cholesterol Education Program Expert Panel on Detection E, Treatment of High Blood Cholesterol in A. Third Report of the National Cholesterol Education Program (NCEP) Expert Panel on Detection, Evaluation, and Treatment of High Blood Cholesterol in Adults (Adult Treatment Panel III) final report. Circulation. 2002;106(25): 3143-3421.

7. European Association for Cardiovascular Prevention and Rehabilitation, Reiner Z, Catapano AL, et al. ESC/EAS Guidelines for the management of dyslipidaemias: the task force for the management of dyslipidaemias of the European Society of Cardiology (ESC) and the European Atherosclerosis Society (EAS). Eur Heart J. 2011;32(14):1769-1818.

8. Stone NJ, Robinson JG, Lichtenstein AH, et al. 2013 ACC/AHA guideline on the treatment of blood cholesterol to reduce atherosclerotic cardiovascular risk in adults: a report of the American College of Cardiology/American Heart Association Task Force on Practice Guidelines. Circulation. 2014;129(25 Suppl 2):S1-S45.

9. Bellosta S, Paoletti R, Corsini A. Safety of statins: focus on clinical pharmacokinetics and drug interactions. Circulation. 2004;109 (23 Suppl 1):III50-III57.

10. Stone NJ, Bilek S, Rosenbaum S. Recent National Cholesterol Education Program Adult Treatment Panel III update: adjustments and options. Am J Cardiol. 2005;96(4A):53E-59E.

11. Baigent C, Landray MJ, Reith C, et al. The effects of lowering LDL cholesterol with simvastatin plus ezetimibe in patients with chronic kidney disease (Study of Heart and Renal Protection): a randomised placebo-controlled trial. Lancet. 2011;377(9784):2181-2192.

12. Waters DD. LDL-cholesterol lowering and renal outcomes. Curr Opin Lipidol. 2015;26(3):195-199.

13. Tonelli M, Wanner C, Kidney Disease: Improving Global Outcomes Lipid Guideline Development Work Group Members. Lipid management in chronic kidney disease: synopsis of the Kidney Disease: Improving Global Outcomes 2013 clinical practice guideline. Ann Intern Med. 2014;160(3):182.
Vascular Health and Risk Management

\section{Publish your work in this journal}

Vascular Health and Risk Management is an international, peerreviewed journal of therapeutics and risk management, focusing on concise rapid reporting of clinical studies on the processes involved in the maintenance of vascular health; the monitoring, prevention and treatment of vascular disease and its sequelae; and the involvement of

\section{Dovepress}

metabolic disorders, particularly diabetes. This journal is indexed on PubMed Central and MedLine. The manuscript management system is completely online and includes a very quick and fair peer-review system, which is all easy to use. Visit http://www.dovepress.com/ testimonials.php to read real quotes from published authors. 